\title{
Is Animal Pain Conscious?
}

\author{
Joseph J. Lynch \\ Cal Poly, San Luis Obispo
}

Editors' Note: This paper by Professor Lynch and the commentary by Professor Duran were presented at the Eastern Division meetings of the Society for the Study of Ethics and Animals, held in Atlanta, Georgia, December, 1993.

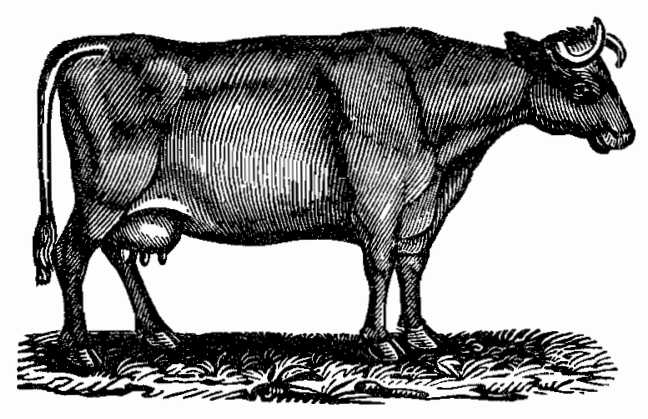

It takes much philosophical resolve to deny that animals feel their pains. After all, the evidence in its favor appears to be overwhelming. As Richard Sergeant observed. Every particle of factual evidence supports the contention that the higher mammalian vertebrates experience pain sensations at least as acute as our own. ${ }^{1}$ The behavioral evidence is indeed very strong: many animals behave just as humans do when in contact with noxious stimuli. Pain-behavior includes not only cries and yelps, but also increased blood pressure, dilated pupils, etc. In addition, many animals have a neurological structure sufficiently like ours, including both $C$ - and A-fibers that serve as pain-transmitters, to warrant the belief that they feel their pains. And finally, as Gareth Matthews has urged, ${ }^{2}$ evolution would lead us to expect apsychological continuity between the species; it would be surprising if among the creatures of the earth only human beings were capable of feeling pain.

Recent arguments by Peter Carruthers ${ }^{3}$ and by Peter Harrison ${ }^{4}$ have resurrected a Cartesian attitude toward animal pain. If their positions are sound, we are mistaken in thinking that animals can feel their pains; and consequently we are also mistaken in thinking that animals could possibly be the appropriate objects of our

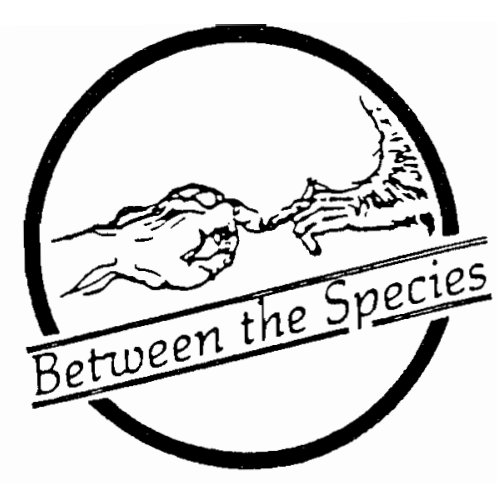

moral sympathies. In part I of this paper, I briefly describe Carruthers' and Harrison's positions. In parts II and III, I show that their Cartesian conclusions are unjustified.

I.

Harrison's and Carruthers' arguments are related but distinct. Harrison acknowledges that the case for animal pain appears strong but argues that critical reflection shows that there is plenty of room for doubt nonetheless. Carruthers claims that his account of conscious experience makes it implausible that nonhuman animals could be conscious of their experience generally, so they can't be conscious of their pain states in spite of apparent evidence to the contrary. Thus, they each contend that the prima facie evidence for animal pain is outweighed by other considerations. Animals engage in painbehavior, they argue, but do not and indeed cannot feel their pains.

Harrison urges that behavioral and neurological evidences are insufficient to establish that a creature can feel its pain. First consider behavioral evidence. Even very simple organisms will withdraw from harmful stimuli, yet few are tempted to attribute conscious pain-states to such organisms. These creatures engage in behavior similar to what we associate with being in pain, but few would attribute conscious pain states to them on the basis of their behavior alone. The

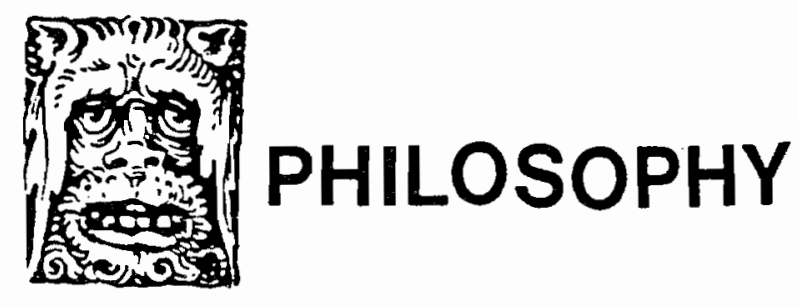


adaptivity of the "pain" of animals, Harrison argues, is thus fully explicable in terms of pain-behavior, taking evasive action with respect to harmful stimuli, without the phenomenological quality of pain.

Harrison further contends that the fact of neurological similarity between humans and animals does not force the attribution of a genuine pain-state. Though many links between the brain and psychological experience are fairly well established, Harrison notes there is a plurality of cases that challenge any straightforward correspondence; so there's always room for doubt. His own suggestion is that consciousness of pain is only properly attributable to rational agents:

...while it is undeniable that animals sense noxious stimuli and react to them, these stimuli only need be represented as unpleasant mental states if they are to become the body's reasons in the context of other reasons. Only as various degrees of unpleasantness can they be taken seriously as ainongst reasons, and this is only necessary in the mind of a rational agent. ${ }^{5}$

The ability to feel one's pain is tied up with the capacity to engage in practical reasoning. One must be able to use pain as among the individual's reasons in deciding what to do. Presumably, animals do not have the stuff of rational agents, so they can't feel their pains on Harrison's view. To be able to feel a pain, apparently, the concept of pain must have a meaning within an individual's cognitive framework. If animals do not feel their pain, animal pain could be classified as "unconscious experience." Harrison refers to Carruthers to support the plausibility of this controversial idea.

Carruthers' contention is that all animal experience is unconscious. While this is certainly a strongly Cartesian-sounding thesis, it is still unlike Descartes in that Carruthers does not deny mental states or processes to nonhuman organisms. ${ }^{6}$ What Carruthers denies is that animals can be reasonably said to be conscious of the mental states that they have. Whereas Descartes took consciousness, or conscious thought, as definitive of mental states, Carruthers recognizes that many mental states are non-conscious. According to him, a conscious state is simply a mental state that is available for thought. ${ }^{7}$ This holds for beliefs as well as for experience.

Carruthers' account is a variation of a standard characterization offered by David M. Armstrong: a mental state $m$ is conscious if it causes a second-order belief in the existence of $m .^{8}$ Thus, a creature might have a belief that plays a causal role in its behavior, but it is a conscious belief only if it also produces the belief in the existence of the first-order belief. Carruthers' account, however, does not demand higher-order beliefs in order to produce conscious states; but since conscious states must be available for thought, every conscious state requires higherorder intentional mentality. And Carruthers takes it as axiomatic that animals could neither have beliefs or thoughts about their experience. ${ }^{9}$

Since a conscious experience is one that can be thought about, and since it is implausible, according to Carruthers, to hold that animals can think about their experience, it follows that they have no conscious experience at all. There is, on this view, nothing that it is like to be a bat or any other nonhuman animal. Carruthers provides additional support for Harrison's controversial suggestion that only thinking beings can be aware of their pains.

If all animal experience is unconscious, then, of course, animal pain is unconscious. ${ }^{10}$ The notion of unconscious pain is initially quite counterintuitive. Indeed consciousness seems to be central to our understanding of experience generally. However, there are common examples from human experience that seem to support it. For example, Rosenthal points out that we are often temporarily distracted from our headaches, and so become at least momentarily unconscious of them. ${ }^{11}$ We don't say, in such cases, that we've had several distinct headaches throughout the day; rather, we seem to acknowledge that we can become temporarily unconscious of the headache pain. ${ }^{12}$

And there are examples, as Carruthers is quick to point out, of perceptual behavior without the usual attendant subjective qualities, such as human blindsightedness. ${ }^{13}$ In such cases, individuals apparently are perceiving, but the perception seems best characterized as unconscious. And, closer to home, there are examples of people engaging in pain-behavior and later reporting they felt no pain, as under the conditions of an intense battle. Pain-behavior, according to Carruthers, requires perception, but perception need not be conscious: organisms can respond to perceptual cues in the environment without ever being aware that they are responding to them.

One might object that if an organism is responding perceptually to cues, then it must be conscious of the 
cues to which it is responding. ${ }^{14}$ Yet, it appears that humans respond unconsciously to perceptual cues regularly. Carruthers cites an example with which most are undoubtedly acquainted: driving an automobile over a familiar route while thinking and even daydreaming about other things. ${ }^{15}$ It may be that one cannot even remember any of the details of the route just driven. But then it appears that there are good grounds for saying that one was unconscious of driving throughout this trip-even though one must have responded to perceptual cues along the way.

The driving example describes a case of unconscious beliefs and desires, not unconscious pain. Nevertheless, the point is that the notion of an unconscious experience is familiar to us. Carruthers acknowledges that while it is part of the normal function of pain to "intrude upon consciousness in order to produce evasive action," an unconscious pain could, in principle, produce the evasive action without any conscious quality or without any conscious desire for the cessation of the pain.

If Carruthers and Harrison are right, then there is some merit in denying that animals can be the objects of moral concern. ${ }^{16}$ By most accounts, the moral standing of animals depends upon their being able to have conscious experience, especially consciousness of pain; for the explanation of why pain is generally regarded as intrinsically bad is that the quality of the subjects' experience is aversive. On both Carruthers' and Harrison's views, animal "experience" has no subjective quality, so mere animal pain-behavior should be no cause for moral concern. It might be asked whether unconscious pain may somehow serve as a suitable basis for moral sympathy. The answer appears to be negative: pity is appropriate only when the object of pity has conscious states. Carruthers makes this clear by imagining a case analogous to blind-sightedness:

Suppose that a particular subject, Mary, is never conscious of any pains in her legs. But when she suffers injury in that region, she displays much of normal pain behavior. If we jab pins into her feet, she tends to try very hard to make us stop, she grimaces and groans, and severe damage causes her to scream. But she sincerely declares that she feels nothing. ${ }^{17}$

Were this case actual, we would surely have grounds for moral pity even though Mary doesn't feel the pain.

\section{Editorial}

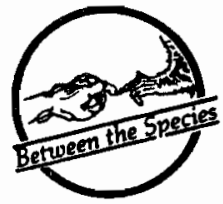

Substantially aided by a $\$ 1,000$ grant from the Animal Protection Institute suggested by Cheryl Mouras, the first issue of Between the Species appeared as "1984 Winter 1985" at Thanksgiving, 1984. Steve and I wanted not to have to produce a second issue almost immediately after the first. Now it is Winter 1995. By the calendar we have completed the first ten years of publishing the journal. In fact, of course, readers will only recently have received Volume 9 Number 4 (Fall, 1993). During most of the years of $B T S$, publication of the journal has been late. And, at times, BTS has appeared to be on the brink of cessation.

In late 1984 Steve bought a new ball for his dot-matrix printer, and dedicated part of his sabbatical year to typing out the articles that would appear in BTS. These he would print out in three inch wide continuous columns, which I would then cut with scissors and strip into pages, afterwards adding the graphics and titles. I still do the graphics by hand, but for many years Rose Lernberg has done the formatting using PageMaker, and hers has at times been a Herculean task. Without her many, many hours of work, it is certain that $B T S$ could not have survived.

Even so, there have been times when the future of the journal was in doubt. At each such time, someone has stepped forward to save the day. Most recently, Professor Harlan Miller and his assistants at Virginia Tech have made it possible for us to get past the impasse that existed a year ago. Professor Miller now scans many of the manuscripts, spell-checking and proofing them also. After the first few years it

(continued on page 80) 
The tragedy of this situation would only seem slightly mitigated by the absence of conscious pain. But as Carruthers urges,

We might perhaps feel sympathy for her general condition, since it is in many ways a disturbing situation in which to find oneself. But we should not feel sympathy on specific occasions of injury, since it is clear that she does not suffer. Not being conscious of any pain, her mental state is not an appropriate object of moral concern. ${ }^{18}$

Carruthers' goes on to point out that the fact that Mary's "suffering" is an indirect consequence of her injury: many of her desires, including the desire to walk freely, would be frustrated. So, Mary is unhappy, and her unhappiness is appropriate for our moral concern. But Mary's unhappiness is caused by her being conscious of her situation. If my car is similarly inoperable, pity would be misdirected at it (though it might well be directed at me) because the car is not conscious of its condition: the car's condition would not cause a frustration of any of its desires, since it has none. On Carruthers' account of consciousness, conscious states, whether of pain or anything else, are supposed to be impossible for animals. And moral pity cannot sensibly be directed towards individuals lacking consciousness. Thus, while moral pity may be appropriate for Mary (since she is conscious of her plight), such pity is not appropriate for a similarly situated non-conscious creature.

On both Carruthers' and Harrison's accounts then, it is the capacity for thought that provides the sole basis for a creature's falling within the scope of morality: a creature counts morally only if it is conscious, and it is conscious only if it can engage in thought. And animals cannot think. Since they cannot think, their pain must be of the unconscious variety. But a creature's unconscious pain is of moral relevance only if, like Mary, the creature has conscious desires not to be in whatever state it is in. The reason that only conscious pain is morally relevant is that it has a quality that individuals generally desire to cease: when we are in pain, we generally seek relief. But unconscious pain has no quality whatsoever. And if a creature is not aware of its situation or aware of its pain, it's hard to see how one could owe any direct obligation to that creature. ${ }^{19}$
II.

While Carruthers and Harrison may draw appropriate moral conclusions, they draw them from erroneous premises. ${ }^{20}$ Their arguments depend largely upon moving from the conceivability of some unconscious pain to the claim that animal pain in general must be unconscious. But the mere possibility that we can account for some pain as a type of unconscious perception is not sufficient to establish that animal pain is most plausibly interpreted in this way.

The strategy employed by Harrison was to take up the considerations in favor of animal pain one by one and show that none implies that animals really feel their pains. While none of the arguments individually establishes that animals have pain, cumulatively they provide very strong support for the thesis. In a criminal investigation, no single piece of evidence typically established the guilt of the accused, but the combined force of evidence may well remove any reasonable doubt. Similarly, behavioral and neurological evidence together with arguments from evolution are sufficiently persuasive to remove all reasonable doubt with regard to the conscious pain of animals. ${ }^{21}$

It is difficult to imagine pain having the survival value that it has for organisms if they were always unconscious of their pain. Carruthers himself acknowledges, part of the normal functional role of pain is that "it gives rise to a conscious desire that the pain should cease." 22 Perhaps it's conceptually possible that pain could exist without this particular causal function, but it seems prima facie unlikely that this could be the norm, given the functional role that pain has for survival. ${ }^{23}$ The process of conditioning is utterly mysterious unless it is presupposed that stimuli can be felt. Indeed, it's hard to make sense of Harrison's acknowledgment that animals "sense noxious stimuli" [my emphasis], if they don't feel. What is this "sensing"? While pain behavior without the attendant consciousness of the pain is clearly imaginable, and in unusual cases has even occurred, the evidence suggests that such cases must be exceptional.

Indeed the examples used to motivate the case for human unconscious perception typically involve the use of other conscious states. For example, once one has learned to drive and has become acquainted with a particular route (note that such learning experiences seem to require the conscious attention of the agent), one can begin to daydream, solve logic problems, or engage in other mental projects while one drives. Most 
human beings are smart enough to carry on this sort of task without paying much attention. Presumably, a less intellectually advanced individual must pay full attention to complete the job successfully. "Unconscious" behavior appears to be more of a case of intelligent cognitive economy within the stream of conscious experiences. Because he does not recognize this, Carruthers confuses being conscious of something with paying attention to something of which one is already conscious. The capability not to take note of one's perceptual experience seems to be best understood as the capacity to shift attention from perceptual experience to other matters. Unconscious behavior therefore, is for the most part a matter of diverting one's attention from certain conscious experiences and focusing on others. When I walk around the campus where I work, I am conscious of many sorts of things, but I focus my attention on just a few. It would just be wrong to say that I am unconscious of those things to which I don't focus my attention. My contention then is that many of the alleged cases of "unconscious perception" could and should be similarly characterized as conscious after all.

Related considerations hold for alleged instances of unconscious pain. Consider the headache example. My attention may well be directed away from my headache, but my headache need not be described as an instance of "unconscious pain" during these momentary distractions. Some might object that this is mere quibbling about how phenomena like the headache ought to be described. ${ }^{24}$ But even if the headache should count as unconscious pain, a headache that never impinged on consciousness would be no headache at all. The behaviors associated with having headaches would be utterly senseless unless there is conscious pain. People typically do not continue to engage in pain-behavior while they are unconscious of or distracted from their pain. And I see no reason why nonhuman animal experience need be any different than ours in this respect. The aversive subjective quality of pain is, after all, part of the functional role that pain plays. This phenomenal quality provides the motivation to move away from dangerous stimuli. Consequently, while Harrison is correct in observing that it is the behavior of the organism that contributes to survival, rather than its subjective states, it is normally difficult to account for pain-behavior in the absence of the experience of pain. In general, so-called unconscious pain is intelligible only in contexts where conscious pain can take place.
III.

What I've tried to show so far is first, that the cumulative argument in favor of conscious animal pain is stronger than either Harrison or Carruthers allow, and second, that many cases of apparent unconscious experience (including pain) are better described as within the stream of consciousness. However these considerations do not address the central contention that any sort of consciousness, including consciousness of pain, must involve the capacity to think, a capacity animals are said to lack. In this section I evaluate this claim.

Recall Harrison's view that since they can't think, animals can't use pain as a reason in deciding what to do. Harrison concentrates on cases in which humans bear pain for other (more heroic) purposes and suggests that if animals can't bear pain for other purposes then they don't feel pain at all. But even if animals could not bear pain for other purposes, it would not follow that they do not feel their pains. There's something very odd about saying that the ability to feel pain depends upon one's ability to count pain as a reason. Indeed the decision to bear pain presupposes that one is conscious of pain, but it's hard to see how the consciousness of pain presupposes any decisions at all. And the fact is that innumerable tests demonstrate that animals will undergo painful stimuli to receive a desired reward. If a creature is initially shocked when attempting to take the food pellet and so refuses the next pellet that is offered, why not say that it counts pain as a reason against retrieving the food pellet? Similarly, if it eventually retrieves the food pellet anyway, hasn't it born the pain for another purpose, just as humans do? Perhaps a stimulus-response story can be told about such cases that does not involve the attribution of any conscious states, though I think it's doubtful; but analogous stories can be told about the human cases as well. Skepticism about the subjective states of others is not limited by species. Humans are surely capable of reasoning with respect to pain and many other things in ways unavailable to most other animals. But, even if humans are "better" thinkers in this sense, it doesn't follow that animals can't think at all.

Carruthers' account of conscious mentality was supposed to render animal consciousness unlikely. It's tempting to maintain that if a theory of consciousness implies something we know to be false-we know animals are sometimes conscious of their pains - then we have a good reason to reject that theory. ${ }^{25}$ But as far 
as I can tell, there's no such implication in Carruthers' own account of consciousness: there's no argument for the implausibility of animals thinking about their experience. ${ }^{26}$ Perhaps Carruthers takes this to be implausible because he has an overly intellectualized notion of just what it is for a creature to think. If we suppose it's true that higher-order mental states are necessary to explain how some states can be conscious, whether we call such higher order states thoughts or beliefs or something else, it is clear that not much mentality is required for an organism to be conscious of its sense-experiences. ${ }^{27}$ That is, a second-order mental state need not involve much cognitive complexity. There is no non-question-begging way to claim that since consciousness necessitates higher-order states, animals cannot be conscious. ${ }^{28}$ If the best theory of consciousness says that consciousness must involve second-order mental states, and the best evidence indicates that animals are conscious, then animals must be capable of at least some higher-order mental states, however modest these may be.

Carruthers shares a widely held philosophical opinion that the capacity to think is dependent upon the capacity to use language. ${ }^{29}$ This is why we are to believe Mary when she tells us that she feels no pain while she engages in pain-behavior. But Mary's case presents us with conflicting evidence as to Mary's sentient states, linguistic behavior and non-linguistic pain behavior, and so it's not easy to decide whether to believe that Mary is in pain. She tells us that she is not, but perhaps her agony has confused her speech. Carruthers assumes that we should believe her words and not her behavior. I see no reason to accept this assumption. And in the case of creatures without language, we don't have to decide between competing evidence in this way. We don't have pain behavior indicating one thing and linguistic behavior indicating another. In the absence, therefore, of evidence to the contrary, the attribution of conscious pain to animals engaging in pain-behavior is normally well-warranted.

Indeed, in many instances of conflict, behavioral evidence could outweigh linguistic. Suppose Mary tells you "I am in pain," but exhibits no other behavioral evidences of pain. The absence of the behavioral evidence might, no doubt, lead us to doubt her words. But the writhing and screams of a one-year old child, or a lamb, is evidence only a philosopher could ignore. Carruthers and Harrison have correctly recognized the centrality of conscious pain for the moral status of animals, but their arguments fall short of showing that animal pain is not conscious. In spite of the recent Cartesian revival, animals remain appropriate objects for moral concern and sympathy.

\section{Notes}

${ }^{1}$ Richard Sergeant, The Spectrum of Pain (London: HartDavis, 1969), p. 72.

2 Gareth Matthews, "Animals and the Unity of Psychology,” Philosophy 53 (1978), pp. 437-453.

3 Peter Carruthers, "Brute Experience," The Joumal of Philosophy 86 (1989), pp. 435-451.

4 Peter Harrison, “Do Animals Feel Pain?' Philosophy 66 (1991), pp. 25-40.

${ }^{5}$ Ibid., p. 37.

${ }^{6}$ Harrison follows Descartes on this point holding that mental states must be conscious.

${ }^{7}$ Carruthers acknowledges a debt to Dennett's "Toward a Cognitive Theory of Consciousness" in Brainstorms (Cambridge, MA: Bradford Books, 1978). Some features of the theory are similar also to David Rosenthal's "Two Kinds of Consciousness" in The Nature of Mind (Oxford: Oxford University Press, 1991) and Peter Smith and O. R. Jones The Philosophy of Mind (New York: Cambridge University Press, 1986).

${ }^{8}$ D. M. Armstrong, The Materialist Theory of the Mind. (London: Routeledge, 1968).

${ }^{9}$ Carruthers believes that his account is an improvement over Armstrong inasmuch as there are instances of the conscious belief that $p$ that do not activate second-order belief that one believes $p$. Each instance of consciousness need not involve a belief that one is conscious. Also, the objects of conscious beliefs are not so much one's inner states; instead conscious beliefs are directed toward the world. Carruthers' criticisms of Armstrong may be off-target. While it may be more precise to explain conscious states in terms of higherorder thoughts, rather than beliefs, one need not assume that the higher-order thought are themselves conscious (otherwise an infinite regress results). See Rosenthal, op. cit.

${ }^{10}$ Against the contention that experience presupposes consciousness, Carruthers describes examples (discussed below) of non-conscious behavior that should be classified as experience since the behavior involved all sorts of psychological states. For related arguments, see Norton Nelkin "Unconscious Sensations," Philosophical Psychology 2 (1989), pp. 129-141.

${ }^{11}$ Rosenthal, op. cit., p. 472. 
${ }^{12}$ See also Searle's discussion in his The Rediscovery of the Mind (Cambridge, MA: M.I.T. Press 1992), pp. 164-167.

13 The phenomena of blind-sightedness occurs when individuals have a damaged visual or striate cortex. In tests, such subjects have been able to correctly guess the objects placed before the affected eye, while reporting that they cannot see the objects. See Weiskrantz "Varieties of Residual Experience," Quarterly Joumal of Experimental Psychology 32 (1980), pp. 365-386, and Natsoulas "Conscious Perception and the Paradox of Blindsight," in G. Underwood, ed., Aspects of Consciousness. (London: Academic Press, 1982).

${ }^{14}$ The blindsighted person does, after all, correctly "guess" the objects placed before the blinded eye. It might be argued that this indicates at least some low-level consciousness of what is perceived.

${ }^{15}$ Carruthers, op. cit., pp. 258-259.

${ }^{16}$ Both Carruthers and Harrison take their arguments as effectively blocking efforts by philosophers defending animal rights or liberation. Both take Peter Singer to task for the scant attention he gives to a defense of animal consciousness in Animal Liberation (New York: Avon Books, 1975) and Practical Ethics (New York: Cambridge University Press, 1979). No doubt Singer, like most of us, thought that the thesis that animals feel their pains requires very little defense. Still, neither Carruthers nor Harrison seem aware of the lengthier defense of animal consciousness offered by Tom Regan in Animal Rights (Berkeley: University of California Press, 1983).

\section{${ }^{17}$ Carruthers, op. cit., p. 267.}

${ }^{18}$ Ibid., p. 267.

${ }^{19}$ In spite of these surprising claims, both Carruthers and Harrison avoid the charge of speciesism. They recognize that infants are, according to their arguments, exempt from conscious pain, and are consequently excluded from any direct moral concern inasmuch as our obligations to them depend upon their present sentient states.

20 But see Jamieson and Bekoff, "Carruthers on Nonconscious Experience," Analysis 52.1 (1992), pp. 26-27 for an argument that moral standing need not depend on conscious experiences. My view on consciousness and moral standing is closer to Carruthers'.

${ }^{21}$ This point is forcefully urged by House, "Harrison on Animal Pain," Philosophy 66 (1991), pp. 366-379.

${ }^{22}$ Carruthers, op. cit., p. $267 \mathrm{n}$.

${ }^{23}$ More precisely, the desire caused by the pain state need not (but can) be conscious, but I contend that the pain state itself must be conscious in order to bring about the appropriate desire whether that desire is conscious or not. I agree with Rosenthal, op. cit., that the requirements for conscious sensory states are much less stiff than those for intentional ones.

${ }^{24}$ See Searle, op. cit., pp. 164-167.

${ }^{25}$ It may be a mistake to analyze consciousness in terms of other non-conscious mental states in this way. While Descartes wrongly excluded the possibility that there are unconscious mental states, consciousness is central in our understanding of mentality. A better account might start with conscious states and analyze non-conscious states in terms of the conscious ones. See Searle, op. cit..

${ }^{26}$ Indeed, Armstrong holds that animal perception does imply animai belief. See Armstrong Belief, Truth and Knowledge (New York: Cambridge University Press, 1973), chapters 1-3.

${ }^{27}$ See Rosenthal, op. cit., p. 472.

${ }^{28}$ An important feature of Rosenthal's account is that a mental state $m$ is conscious only if it gives rise to state $\mathrm{m}$ '. But this does not require that $m^{\prime}$ is conscious as well. Carruthers mistakenly assumes that the higher-order states must be conscious. Not only is there no need for the higher state to be conscious, it is necessary that at least some higherorder states are nonconscious (on pain of infinite regress if higher-order states).

${ }^{29}$ Compare Davidson's "Thought and Talk," in Inquires Into Truth and Interpretation (New York, Oxford, 1984) as well as Malcolm's "Thoughtless Brutes," Proceedings and Addresses of the American Philosophical Association 46 (1973), pp. 5-19). Carruthers position is that the relationship between consciousness and language is contingent.

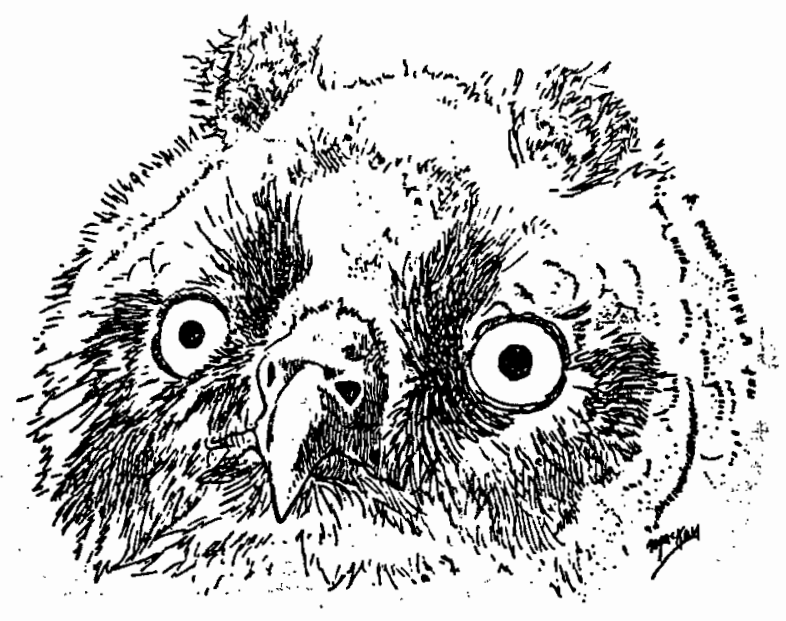

